\title{
A Standard Intervention Practice to Promote Appropriate Lamotrigine Therapy by Pharmacists
}

\author{
Hiroshi Shimamura, ${ }^{*, a, b}$ Hiroko Yamada, ${ }^{a, c}$ Natsumi Okada, ${ }^{c, d}$ Takeshi Uchikura, ${ }^{c}$ \\ Katsumi Tanaka, ${ }^{d}$ Tadanori Sasaki, ${ }^{c, d}$ and Hiroyuki Itabe ${ }^{b}$ \\ ${ }^{a}$ Department of Pharmacy, Showa University Hospital East Branch; 2-14-19 Nishinakanobu, Shinagawa-ku, Tokyo \\ 142-0054, Japan: ${ }^{b}$ Division of Biological Chemistry, Department of Molecular Biology, Showa University, School \\ of Pharmacy; 1-5-8 Hatanodai, Shinagawa-ku, Tokyo 142-8555, Japan: ${ }^{c}$ Department of Hospital Pharmaceutics, \\ Showa University, School of Pharmacy; 1-5-8 Hatanodai, Shinagawa-ku, Tokyo 142-8555, Japan: and ${ }^{d}$ Department \\ of Pharmacy, Showa University Hospital; 1-5-8 Hatanodai, Shinagawa-ku, Tokyo 142-8666, Japan.
}

Received August 31, 2017; accepted January 4, 2018

The dosage and frequency of lamotrigine administration for each patient must be prescribed carefully according to the disease, age, concomitant medications, and administration period. According to Pharmaceuticals and Medical Devices Agency (PMDA) reports, either the number of patients with adverse events caused by the inappropriate use of lamotrigine or the ratio of the patients who have benefited from the Relief System for Adverse Drug Reactions did not change even after the revision of the package insert in 2015. The procedure for auditing lamotrigine prescriptions was standardized to improve patient safety in our hospitals. The efficacy of standardization was evaluated by investigating the patients' records and prescriptions. A total of 77 patients treated with lamotrigine were examined to evaluate the appropriateness of the dosage and frequency of administration as well as the number of prescription enquiries made by pharmacists retrospectively. In addition, the presence of adverse events such as skin rash was examined using medical records. The number of inappropriate cases found and the prescription questions asked by pharmacists during the 24-month period before and after standardization were compared. The rate of inappropriate prescriptions after standardization was significantly lower than that before $(12.1 \mathrm{vs} .29 \% ; p<0.05)$. The rate of prescription questions raised after standardization was significantly higher than that before $(37.0 v s .1 .7 \% ; p<0.05)$. Moreover, there was no adverse event after standardization. Our standardization practice was effective to easily identify inappropriate prescriptions and provide physicians with the appropriate dosage or frequency of administration for the patients.

Key words lamotrigine; appropriate use; prescription question; audit; skin rash; adverse event

Lamotrigine is an anticonvulsant used for the treatment of patients with epilepsy and bipolar disorder. The mechanism of action of this drug relates to inhibition of glutamate release (an excitatory amino acid), inhibition of voltage-sensitive sodium channels, and stabilization of neuronal membranes. ${ }^{1,2)}$ The dosage and frequency of lamotrigine administration vary according to the patient's specific disease, age, concomitant medications, and administration period. Sodium valproate (VPA) increases the serum concentration of lamotrigine by inhibiting its glucuronidation; therefore, the dosage of lamotrigine for patients using VPA should be reduced. Conversely, concomitant medications, including carbamazepine and phenytoin, induce lamotrigine glucuronidation. Because these medications decrease the serum concentration of lamotrigine, the dosage of lamotrigine for patients using them should be increased.

It is highly complex for physicians and pharmacists to evaluate the dosage and administration of this medication, considering all of these conditions. In addition, pharmacists may not always identify questionable prescriptions involving lamotrigine because it is difficult to specify which part of the prescription is inappropriate.

Lamotrigine can cause serious rashes requiring hospitalization and discontinuation of treatment. The majority cases of life-threatening rashes associated with lamotrigine have occurred within 2 to 8 weeks after treatment started. The risk of serious rashes increases when VPA is coadministrated with lamotrigine and the lamotrigine dosage exceeds recommended initial doses, recommended initial dose titration, or the recommended dose escalation for lamotrigine. ${ }^{1,2)}$ A phase III clinical trial of lamotrigine carried out in Japan reported that $10.4 \%$ (18/173) of outpatients who were prescribed a combination of lamotrigine and VPA suffered from a skin rash when they were administered a higher-than-approved dosage. ${ }^{3)}$ In contrast, the incidence of skin rash was found in $2.9 \%(3 / 102)$ of patients who were prescribed lamotrigine and VPA at an approved dosage. ${ }^{3,4)}$ The patients who suffer from a severe skin rash are supported by the "Relief System for Adverse Drug Reactions" in Japan. However, this institution will not support a patient when lamotrigine is prescribed inappropriately. ${ }^{5,6)}$ Therefore, there is a social need for improved quality of lamotrigine therapy. The Japanese Society of Hospital Pharmacists issued a notification for pharmacists regarding the appropriate use of lamotrigine." The package insert for lamotrigine was revised in September 2015, immediately after this study period; this revision enabled pharmacists to easily understand the differences in the dosage and frequency of lamotrigine administration according to the patient's disease, age, concomitant medications, and administration period. However, the number of patients with adverse events due to inappropriate use of lamotrigine or the ratio of patients who received benefits from the Relief System for Adverse Drug Reactions did not change [40 out of 61 (65.6\%), Sep 2015-Aug 2016 vs. 47 out of 74 (63.5\%), Sep 2014-Aug 2015] according to the reports from Pharmaceuticals and Medical Devices 
Agency (PMDA). ${ }^{89}$ Although patients may be suffering from severe adverse events, they cannot currently be supported by the Relief System for Adverse Drug Reactions.

In general, prescribing faults can arise from many steps including the choice of drug, the dosage, the administration and frequency, but errors in dose selection occur most commonly that represent $>50 \%$ of all prescribing faults. ${ }^{10)}$ In a retrospective study of outpatient prescriptions of new anti-epileptic drugs, Murphy et al. reported that pharmacist intervention was required in as much as $17 \%$ of the prescriptions (44 out of 260 prescriptions). Fifteen out of the 44 prescriptions of antiepileptic drugs (34\%) contacted by pharmacists were due to an error in dose or frequency of administration. ${ }^{11)}$ Interventions by ward-based pharmacists were shown to be effective in reducing drug-related readmissions. ${ }^{12)}$ Therefore, pharmacists could have a role in a systematic audit for the prescriptions of lamotrigine.

In this study, the procedure for auditing lamotrigine prescriptions was standardized in our hospital to ensure that all pharmacists can question inappropriate prescriptions. Our procedure requires patient's specific disease, age, concomitant medications, and administration period of lamotrigine from the clinical record. The efficacy of standardization practice for patient safety was evaluated by examining patient prescriptions.

\section{METHODS}

Standardization of Procedures for Auditing Lamotrigine Prescriptions Since the package insert for lamotrigine did not described systematically in 2013 at the timing we started
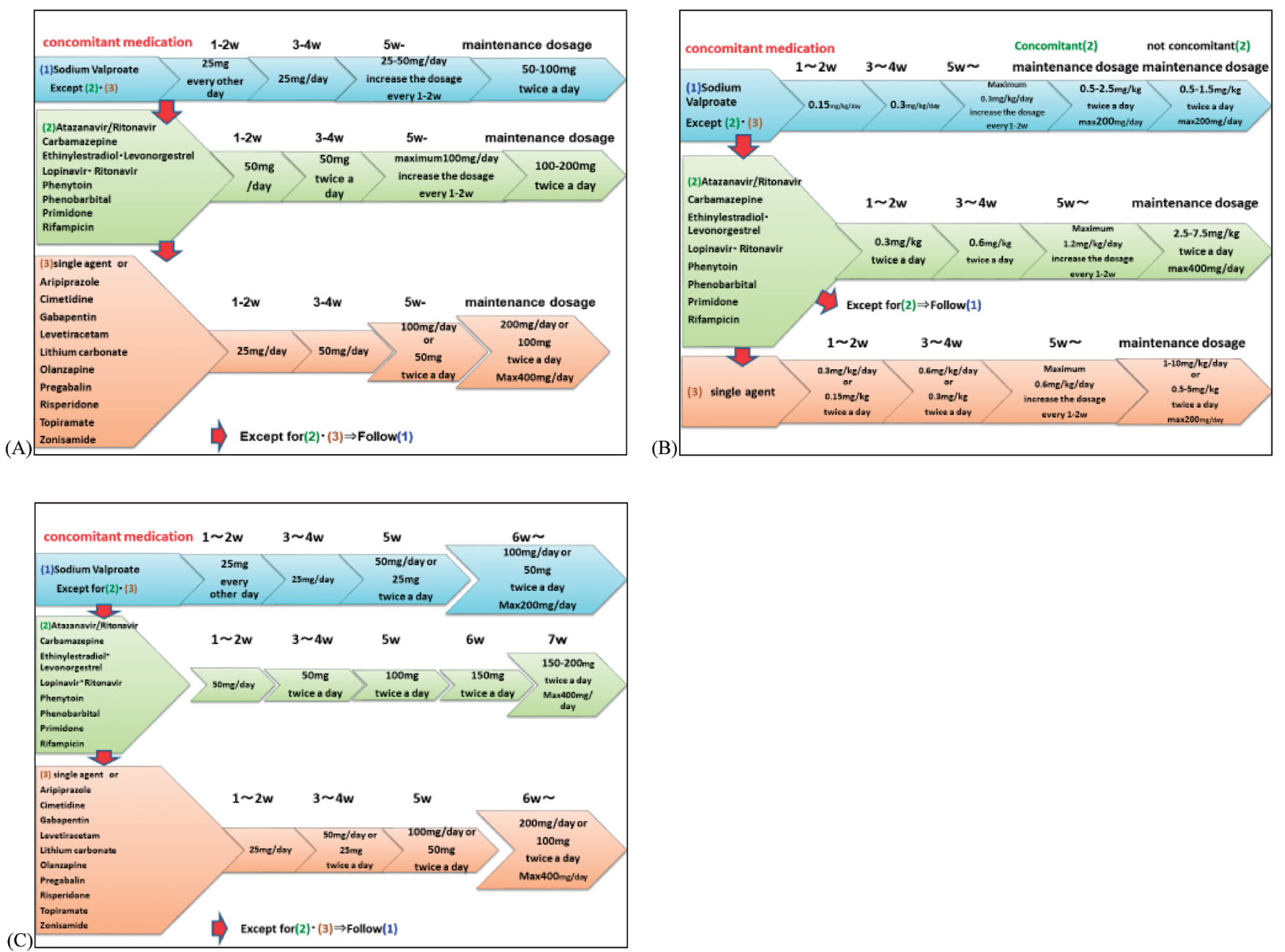

Fig. 1. A Set of Charts Used to Evaluate Lamotrigine Prescriptions

(A) Chart A to check prescriptions containing lamotrigine (for adult patients with epilepsy). This chart describes the appropriate dosage and administration for patients aged $\geq 16$ years and with epilepsy. Top arrows (1) should be followed when lamotrigine is prescribed with VPA. Middle arrows (2) should be followed when VPA is not prescribed, but one of the other eight medications listed is used. Bottom arrows (3) should be followed when neither VPA nor the other eight medications in the middle arrows are prescribed, but one of 10 other medications is used as a concomitant medication. Top arrows should be followed when the middle and bottom arrows are not applicable. By following one of these arrow trails, we can find the appropriate dosage for the specified administration period. (B) Chart B to check the prescriptions containing lamotrigine (for child patients with epilepsy). This chart is applicable for patients aged $<16$ years and with epilepsy. Top arrows (1) should be followed when VPA is prescribed for the patient. Middle arrows (2) should be followed when VPA is not prescribed, but one of the other eight medications listed is used. Bottom arrows (3) should be followed when any concomitant medication is not prescribed. Top arrows should be followed when the middle arrows are not applicable. By following one of these arrow trails, we can identify the appropriate dosage for the specified administration period. When one of the eight medications (2) is prescribed with VPA, the maintenance dosage is $0.5-2.5 \mathrm{mg} / \mathrm{kg}$ twice a day (maximum: $200 \mathrm{mg} / \mathrm{d}$ ). When none of the eight medications (2) is prescribed alongwith VPA, the maintenance dosage is $0.5-1.5 \mathrm{mg} / \mathrm{kg}$ twice a day (maximum: $200 \mathrm{mg} / \mathrm{d}$ ). (C) Chart $\mathrm{C}$ to check prescriptions containing lamotrigine (for patients with bipolar disorder). This chart is used for patients with bipolar disorder. Top arrows (1) should be followed when VPA is prescribed with lamotrigine. Middle arrows (2) should be followed when VPA is not prescribed, but one of the other eight medications listed is used. Bottom arrows (3) should be used when neither VPA nor any of the other eight medications in the middle arrows is prescribed, but any of the 10 other medications is used as a concomitant medication. Top arrows should be followed when the middle and bottom arrows are not applicable. By following one of these arrow trails, we can identify the appropriate dosage for the specified administration period. 
this study, we made charts to audit lamotrigine prescriptions. Any conditions, restrictions, and recommendations regarding lamotrigine, including the pharmacokinetics and interaction with other medications, as well as the dosage and frequency of administration described in the package inserts were extracted. These conditions were organized and classified, and charts supporting the appropriate use of lamotrigine were prepared (Fig. 1).

The charts were introduced into the computerized ordering system of Showa University Hospitals on July 1, 2013, and the audit process was standardized. Since then, the charts have been used as a reference when a prescription containing lamotrigine is being audited.

Evaluation of Standardized Procedures for Audit Purposes This retrospective observational study was performed at Showa University Hospital and Showa University Hospital East Branch over the course of four years from July 1, 2011 to June 30, 2015. The study included 77 hospitalized patients with epilepsy or bipolar disorder who were prescribed lamotrigine. Patients with other diseases were excluded in this study. The 423 prescriptions issued during the study period were examined regarding the appropriateness of the dosage and frequency of administration as well as the presence of prescription enquiries made by pharmacists. Moreover, the presence of a skin rash as an adverse event was examined using the patient's medical records. The number of inappropriate cases identified and prescription questions asked by pharmacists during the 24-month study period before and after standardization were compared (July 1, 2011-June 30, 2013 vs. July 1, 2013-June 30, 2015). Using the charts we inspected appropriateness of the all prescriptions containing lamotrigine. Prescriptions that followed the package insert were defined as appropriate, and those did not match either the dosage or frequency of administration were defined as inappropriate. Patient information was anonymously extracted from clinical records. The research protocol was approved by the Human Ethical Committee of Showa University, School of Pharmacy (Approval number 235).

Statistical Considerations Fisher's exact test was used to compare the categorical variables. A $p$-value of $\leq 0.05$ was considered significant. All statistical analyses were performed using EZR software (Saitama Medical Center, Jichi Medical University, Japan), which is a graphic user interface for R (The R Foundation for Statistical Computing, version 2.13.0). ${ }^{13)}$ More precisely, it is a modified version of $\mathrm{R}$ Commander (version 1.6-3) that includes statistical functions frequently used in biostatistics.

\section{RESULTS}

To evaluate the usefulness of the standardized procedure for a total of 77 patients, 423 prescriptions containing lamotrigine in the computerized physician order entry system were retrospectively examined. The characteristics of the study population are shown in Table 1. The total numbers of patients before and after implementing standardization were 33 and 44, respectively. There were 25 adults $(75.8 \%)$ and eight children $(24.2 \%)$ in the before standardization group, and 34 adults (77.3\%) and 10 children $(22.7 \%)$ in the after standardization group. There were $22(66.7 \%)$ patients with epilepsy and 11 $(33.3 \%)$ with bipolar disorder in the before standardization
Table 1. Patient Characteristics

\begin{tabular}{lccc}
\hline \hline & Jul $2011-$ & Jul $2013-$ & \\
& Jun $2013(n=33)$ & Jun $2015(n=44)$ & \\
& & & \\
\hline Age & $25(75.8 \%)$ & $34(77.3 \%)$ & 1 \\
$\geq 16$ years & $8(24.2 \%)$ & $10(22.7 \%)$ & \\
$<16$ years & & & \\
Diseases & $22(66.7 \%)$ & $22(50.0 \%)$ & 0.17 \\
Epilepsy & $11(33.3 \%)$ & $22(50.0 \%)$ & \\
Bipolar disorder & & & 1 \\
Concomitant medications & $14(42.4 \%)$ & $16(36.4 \%)$ & 0.64 \\
$(1)$ & $6(18.2 \%)$ & $7(15.9 \%)$ & 1 \\
$(2)$ & $6(18.2 \%)$ & $18(40.9 \%)$ & $<0.05$ \\
$(3)$ & $7(21.2 \%)$ & $3(6.8 \%)$ & 0.06 \\
Others & & & \\
Administration period & $9(27.3 \%)$ & $8(18.2 \%)$ & 0.41 \\
Induction & $24(72.3 \%)$ & $36(81.8 \%)$ & \\
Maintenance & & & \\
Hospitals started lamotrigine & $23(69.7 \%)$ & $30(68.2 \%)$ & 1 \\
Our hospitals & $10(30.3 \%)$ & $14(31.8 \%)$ & \\
Others & & & \\
\hline
\end{tabular}

$n$ : number of patients. Concomitant medications. (1): VPA. (2): Atazanavir/Ritonavir, Carbamazepine, Ethinylestradiol/Levonorgestrel, Lopinavir/Ritonavir, Phenytoin, Phenobarbital, Primidone, Rifampicin. (3): Aripiprazole, Cimetidine, Gabapentin, Levetiracetam, Lithium carbonate, Olanzapine, Pregabalin, Risperidone, Topiramate, Zonisamide.

Table 2. Number of Appropriate and Inappropriate Prescriptions Containing Lamotrigine

\begin{tabular}{|c|c|c|c|}
\hline $\begin{array}{l}\text { Appropriateness of } \\
\text { prescription }\end{array}$ & $\begin{array}{c}\text { Jul 2011- } \\
\text { Jun } 2013(n=200)\end{array}$ & $\begin{array}{c}\text { Jul 2013- } \\
\text { Jun } 2015(n=223)\end{array}$ & $p$-Value \\
\hline Appropriate & $142(71.0 \%)$ & $196(87.9 \%)$ & $<0.05$ \\
\hline Inappropriate & $58(29.0 \%)$ & $27(12.1 \%)$ & \\
\hline
\end{tabular}

$n$ : number of prescriptions.

Table 3. Number of Questions Asked by Pharmacists Regarding Prescriptions Containing Lamotrigine

\begin{tabular}{cccc}
\hline \hline Responses & $\begin{array}{c}\text { Jul 2011- } \\
\text { Jun 2013 }(n=58)\end{array}$ & $\begin{array}{c}\text { Jul 2013- } \\
\text { Jun 2015 }(n=27)\end{array}$ & $p$-Value \\
\hline $\begin{array}{c}\text { Asked prescription } \\
\text { questions }\end{array}$ & $1(1.7 \%)$ & $10(37.0 \%)$ & $<0.05$ \\
$\begin{array}{c}\text { Not ask prescription } \\
\text { questions }\end{array}$ & $57(98.3 \%)$ & $17(63.0 \%)$ & \\
\hline
\end{tabular}

$n$ : number of inappropriate prescriptions.

group, and 22 (50\%) with epilepsy and 22 (50\%) with bipolar disorder in the after standardization group.

The number of inappropriate prescriptions after standardization was significantly lower than that before standardization [27 (12.1\%) vs. 58 (29.0\%); $p<0.05]$ (Table 2). The number of prescription questions raised by pharmacists after standardization was significantly higher than that before standardization [10 (37.0\%) vs. 1 (1.7\%); $p<0.05]$ (Table 3). The details of inappropriate prescriptions included underdose, overdose, early increase of the dosage, and off-label use (Table 4). There were some cases of off-label use wherein lamotrigine was prescribed without any concomitant medication. The use of lamotrigine as a single agent was not approved as of 2013. The number of cases was reduced after standardization (induction at our hospitals) for all the categories except overdose 
Table 4. Details of Inappropriate Prescriptions

\begin{tabular}{|c|c|c|c|c|c|c|}
\hline \multirow{2}{*}{$\frac{\text { Induction }}{\text { Problems with the prescriptions }}$} & \multicolumn{3}{|c|}{ Our hospitals } & \multicolumn{3}{|c|}{ Other hospitals } \\
\hline & $\begin{array}{c}\text { Jul 2011-Jun } 2013 \\
\qquad(n=37)\end{array}$ & $\begin{array}{c}\text { Jul 2013-Jun } 2015 \\
(n=16)\end{array}$ & $p$-Value & $\begin{array}{l}\text { Jul 2011-Jun } 2013 \\
\qquad(n=21)\end{array}$ & $\begin{array}{c}\text { Jul 2013-Jun } 2015 \\
\qquad(n=11)\end{array}$ & $p$-Value \\
\hline Underdose & 19 & 5 & & 17 & 7 & \\
\hline Initial & $4(10.8 \%)$ & $1(6.2 \%)$ & 1 & 0 & 0 & - \\
\hline Maintenance & $15(40.5 \%)$ & $4(25.0 \%)$ & 0.36 & $17(81.0 \%)$ & $7(63.6 \%)$ & 0.40 \\
\hline Overdose & 9 & 8 & & 1 & 1 & \\
\hline Initial & $9(24.3 \%)$ & 0 & $<0.05$ & 0 & 0 & - \\
\hline Maintenance & 0 & $8(50.0 \%)$ & $<0.05$ & $1(4.8 \%)$ & $1(9.1 \%)$ & 1 \\
\hline Early increase of dosage & $6(16.2 \%)$ & $3(18.8 \%)$ & 1 & 0 & 0 & - \\
\hline Off-label use (single agent*) & $3(8.1 \%)$ & 0 & 0.55 & $3(14.3 \%)$ & $3(27.3 \%)$ & 0.39 \\
\hline
\end{tabular}

* Single-agent use of lamotrigine was not approved as of 2013. $n$ : number of inappropriate prescriptions.

Table 5. Cases for Which Pharmacists Did Not Ask Prescription Questions (Jul 2013-Jun 2015)

\begin{tabular}{|c|c|c|c|}
\hline & $\begin{array}{l}\text { Total } \\
(n=17)\end{array}$ & $\begin{array}{l}\text { Underdose (maintenance dosage) } \\
\qquad(n=8)\end{array}$ & $\begin{array}{l}\text { Overdose (maintenance dosage) } \\
\qquad(n=9)\end{array}$ \\
\hline Continuation of bringing medications $* 1$ & 7 & 4 & 3 \\
\hline (Induction at our hospitals) & $3(17.6 \%)$ & $1(12.5 \%)$ & $2(22.2 \%)$ \\
\hline (Induction at other hospitals) & $4(23.5 \%)$ & $3(37.5 \%)$ & $1(11.1 \%)$ \\
\hline Continuation of previous medications $* 2$ & $8(47.1 \%)$ & $3(37.5 \%)$ & $5(55.6 \%)$ \\
\hline Others & $2(11.8 \%)$ & $1(12.5 \%)$ & $1(11.1 \%)$ \\
\hline
\end{tabular}

$n$ : number of prescriptions that were not questioned. ${ }^{* 1}$ : For patients had been were taken care by other hospitals or by our hospitals as outpatients, cases were prescribed as before when newly admitted to our hospitals. $*^{2}$ : For patients had been hospitalized in our hospitals and prescribed more than twice, cases were prescribed as before.

(maintenance dosage). No patients with severe adverse effects was observed after the standardization, while one patient from the before standardization group who was administered an overdose at the initial administration was suspected of having lamotrigine-induced skin rash. There were 17 cases for which prescription questions were not asked by pharmacists after standardization was implemented. The major reasons for this included continuation of bringing medications (41.2\%) and continuation of previous medications $(47.1 \%)$. Other reasons included cases in which pharmacists asked other prescription questions, but not about lamotrigine (11.8\%) (Table 5).

\section{DISCUSSION}

Our present study demonstrated that introducing a standardized chart to support the appropriate use of a complicated medication into the ordering system was useful for the improvement of lamotrigine administration. It is remarkable that the ratio of inappropriate prescriptions decreased and that of prescription questions asked by pharmacists increased. Therefore, the chart is useful for both physicians and pharmacists in the dispensary room to ascertain the appropriate dosage and perform an audit of the prescription. The chart may help the pharmacists identify inappropriate prescriptions by clarifying only four checkpoints (age, disease, concomitant medications, and initial administration date) and provide the physicians with information regarding the appropriate dosage or frequency for patients.

Skin rashes, including Stevens-Johnson syndrome (SJS) and toxic epidermal necrolysis (TEN), are major adverse events induced by lamotrigine. ${ }^{14,15)}$ The incidences of SJS and TEN caused by lamotrigine have been reported in Japan, and the information has been issued by PMDA. ${ }^{16}$ ) The mortality rates of SJS and TEN during 2004-2010 were approximately
$5 \%$ and $30 \%$ respectively, and aftereffects might be left to the eyes even if they recover. ${ }^{17)}$ The risk of a patient developing a skin rash may increase due to co-administering lamotrigine with VPA, ${ }^{18-20}$ ) exceeding the recommended initial dose of lamotrigine, ${ }^{20,21)}$ or exceeding the recommended increasing dose of lamotrigine. ${ }^{20,22)}$ It is important to check initial dose and dose-titration periods. In the patients who received induction lamotrigine therapy at our hospitals, the number of overdose cases at the time of the initial dosage was reduced from nine to zero. And the number of early increase of the dosage cases was reduced from six to three. The early increase of the dosage cases did not become zero, but pharmacists asked prescription questions in the all three cases after the standardization. There was one patient with a suspected skin rash before standardization was implemented. Although the initial dosage was an overdose, pharmacists did not question the physician about the dosage. It is regrettable that the pharmacists could have proposed the appropriate dosage and administration in this situation. There was no patient with a suspected skin rash after standardization.

The package insert for lamotrigine was revised in September 2015, immediately after this study period; this revision enabled pharmacists to easily understand the differences in the dosage and frequency of lamotrigine administration according to the patient's disease, age, concomitant medications, and administration period. However, the number of patients with severe skin rashes due to inappropriate use of lamotrigine or the ratio of patients who received benefits from the Relief System for Adverse Drug Reactions did not reduce after the revision as reported by PMDA. The patients suffering from severe adverse events cannot currently be supported by the Relief System for Adverse Drug Reactions. ${ }^{8,9)}$ We speculate that an appropriate lamotrigine therapy may not be practiced by only 
referring to the package insert; cooperative operation between physicians and pharmacists may also be critically important. The standardization of procedures for auditing of lamotrigine prescription would improve the quality of medication at any hospital and is highly beneficial for the patients.

After the standardized chart was introduced, the number of inappropriate prescriptions decreased, with some exceptions. The charts were introduced into the computerized ordering system, but physicians must click on their computers to open the drug information page of lamotrigine to refer to the charts. Therefore, the chart is not referred every time the physicians order lamotrigine. This is a limitation of our current system because we do not have an automatic alert function. Therefore, pharmacists should know when inappropriate prescriptions are likely to occur and make the physicians aware of referring to the chart when prescribing lamotrigine.

Although the audit was standardized, there were occasions when pharmacists did not ask questions for inappropriate prescriptions; major reasons for this included continuation of bringing medications and continuation of previous medications. The continuation of bringing medications cases at our hospitals included those with first prescriptions of the maintenance dosage that was over- or underdose. Pharmacists checked the prescription information before admission of these patients, considering that the previous prescriptions were appropriate, and then proceeded without asking questions. The continuation of previous medications cases in our hospitals included those with second to fourth prescriptions of the maintenance dosage that was over- or underdose. When pharmacists observed that the dosage and frequency of lamotrigine had not changed, they proceeded without asking questions. When patients were prescribed lamotrigine before admission in our hospitals, and when an inpatient was continuously prescribed the same dosage and administration, it was difficult for the pharmacists to identify inappropriate prescriptions. In particular, pharmacists would not be able to determine whether a maintenance dose is appropriate when it is underdose. Measuring serum concentrations may be helpful to determine whether the dosage is appropriate. ${ }^{23)}$

It is very important and practical that both pharmacists and physicians understand the major risks of inappropriate prescriptions. Awareness among hospitals might be the first step toward changing pharmacists' behaviors.

\section{CONCLUSION}

The standardization practice may help to easily identify inappropriate prescriptions and provide physicians with the appropriate dosage or frequency of administration for the patients.

Conflict of Interest The authors declare no conflict of interest.

\section{REFERENCES}

1) Fitton A, Goa KI. Lamotrigine. An update of its pharmacology and therapeutic use in epilepsy. Drugs, 50, 691-713 (1995).

2) Goldsmith DR, Wagstaff AJ, Ibbotson T, Perry CM. Lamotrigine: a review of its use in bipolar disorder. Drugs, 63, 2029-2050 (2003).

3) Ohtahara S, Fujiwara T, Kaneko S. lijima M. Clinical evaluation of lamotrigine with the current recommended dose in overseas-phase III study of lamotrigine in patients with epilepsy treated with valproate. New Rem Clin., 57, 1442-1453 (2008).

4) "Pharmaceuticals and Medical Devices Safety Information No. 287.": 〈http://wwwl.mhlw.go.jp/kinkyu/iyaku_j/iyaku_j/anzenseijyouhou/ 287.pdf〉, cited 7 Aug. 2017.

5) Yamada K. Optimization of pharmacotherapy and relief system for adverse drug reaction. Jpn J Clin Psychiatr., 42, 227-234 (2013).

6) "Pharmaceuticals and Medical Devices Safety Information No. 296.": 〈http://www1.mhlw.go.jp/kinkyu/iyaku_j/iyaku_j/anzenseijyouhou/ 296-1.pdf>, cited 7 Aug. 2017.

7) Japanese Society of Hospital Pharmacists.: 〈http://www.jshp.or.jp/ cont/13/0418.pdf, cited 7 Aug. 2017.

8) Pharmaceuticals and Medical Devices Agency.: 〈http://www.pmda. go.jp/relief-services/adr-sufferers/0013.html〉, cited 7 Aug. 2017.

9) Pharmaceuticals and Medical Devices Agency.: 〈http://www.pmda. go.jp/relief-services/adr-sufferers/0036.html, cited 7 Aug. 2017.

10) Velo GP, Pietro Minuz P. Medication errors: prescribing faults and prescription errors. Br. J. Clin. Pharmacol., 67, 624-628 (2009).

11) Murphy AP, Bentur H, Dolan C, Bugembe T, Gill A, Appleton R. Outpatient anti-epileptic drug prescribing errors in a children's hospital: an audit and literature review. Seizure, 23, 786-791 (2014).

12) Gillespie U, Alassaad A, Henrohn D, Garmo H, Hammarlund-Udenaes M, Toss H, Kettis-Lindblad Å, Melhus H, Mörlin C. A comprehensive pharmacist intervention to reduce morbidity in patients 80 years or older. Arch. Intern. Med., 169, 894-900 (2009).

13) Kanda Y. Investigation of the freely available easy-to-use software 'EZR' for medical statistics. Bone Marrow Transplant., 48, 452-458 (2013).

14) Wong ICK, Mawer GE, Sander JWAS. Adverse event monitoring in lamotrigine patients: a pharmaco-epidemiologic study in the United Kingdom. Epilepsia, 42, 237-244 (2001).

15) Rzany B, Correia O, Kelly JP, Naldi L, Auquier A, Stern R; Study Group of the International Case Control Study on Severe Cutaneous Adverse Reactions. Risk of Stevens-Johnson syndrome and toxic epidermal necrolysis during first weeks of antiepileptic therapy: a case-control study. Lancet, 353, 2190-2194 (1999).

16) Pharmaceuticals and Medical Devices Agency.: 〈https://www.pmda. go.jp/files/000198343.pdf), cited 7 Aug. 2017.

17) Sudo C, Azuma Y, Maekawa K, Kaniwa N, Sai K, Saito Y. Current movements of four serious adverse events induced by medicinal drugs based on spontaneous reports in Japan. Bull. Natl. Inst. Health Sci., 129, 111-117 (2011).

18) Fujiwara T. Sinkitenkanyaku wo mochiita tenkan no yakubutsuchiryou guideline. J. Jpn. Epil. Soc., 28, 48-65 (2010).

19) Yalçin B, Karaduman A. Stevens-Johnson syndrome associated with concomitant use of lamotrigine and valproic acid. J. Am. Acad. Dermatol., 43, 898-899 (2000).

20) Guberman AH, Besag FMC, Brodie MJ, Dooley JM, Duchowny MS, Pellock JM, Richens A, Stern RS, Trevathan E. Lamotrigineassociated rash: risk/benefit considerations in adults and children. Epilepsia, 40, 985-991 (1999).

21) Wong ICK, Mawer GE, Sander JWAS. Factors influencing the incidence of lamotrigine-related skin rash: results of a case record survey. Ann. Pharmacother., 33, 1037-1042 (1999).

22) Pharmaceuticals and Medical Devices Agency.: «http://www.pmda. go.jp/files/000145676.pdf), cited 7 Aug. 2017.

23) Patsalos PN, Berry DJ, Bourgeois BF, Cloyd JC, Glauser TA, Johannessen SI, Leppik IE, Tomson T, Perucca E. Antiepileptic drugsbest practice guidelines for therapeutic drug monitoring: A position paper by the subcommission on therapeutic drug monitoring, ILAE Commission on Therapeutic Strategies. Epilepsia, 49, 1239-1276 (2008). 